\title{
Student attitudes in a new hybrid design-based introductory physics laboratory
}

\author{
Katherine Ansell, and Mats Selen \\ University of Illinois at Urbana-Champaign, Physics, \\ 1110 W Green St, Urbana, IL, USA
}

\begin{abstract}
"Cookbook" style introductory physics laboratories are convenient for large-enrollment courses, but evidence indicates that this style of instruction provides little benefit to students. At the University of Illinois, we have piloted a design-based laboratory format in an introductory calculus-based mechanics course. This format emphasizes scientific skills training over conceptual instruction, following the Investigative Science Learning Environment (ISLE) framework. Students in this laboratory pilot were assigned dorm room prelab experiments to be done and submitted online, and worked on design experiments in class. In this paper, we describe the new laboratory format and present preliminary results indicating that this format of instruction improved attitudes toward laboratory classes without impacting overall exam scores.
\end{abstract}

\section{INTRODUCTION}

Introductory physics laboratories are currently undergoing a period of questioning and reform, spurred by the concurrence of two factors: (1) Growing availability of portable high quality data collection technologies and (2) a growing body of evidence in Physics Education Research (PER) questioning the effectiveness of the laboratory as a conceptual physics instruction tool, most recently shown by Wieman and Holmes in [1]. Several universities are moving toward design-based laboratory models to engage students in scientific skill development rather than focusing mainly on a list of physics concepts.

At the University of Illinois, we are redesigning the laboratory portion of our introductory physics curriculum to replace our current "cookbook" activities with design tasks. The aim of this new format is to engage and train students in scientific skills, based on the abilities detailed in the Investigative Science Learning Environment (ISLE) framework [2]. To ameliorate classroom time constraints, we have introduced an online prelab component in which students do preparatory experiments in their dorm rooms, receive feedback via email, and continue to ISLE-inspired design tasks in the classroom.

In the Spring 2016 semester, we implemented a pilot of our IOLab hybrid laboratory in a large-enrollment calculusbased introductory mechanics course. Students in selected sections of the course were instructed using the new curriculum. In this paper, we report the details of this laboratory course structure and show preliminary results comparing the effect of the new and old instruction on our students' attitudes and performance on exams.

\section{A. IOLab hybrid labs}

The University of Illinois offers several large-enrollment (500-1300 student) introductory physics courses at the algebra and calculus level, which include laboratory components. Reforms by the UIUC PER group detailed in [3] describe the research-validated methods that have been used to maintain instructional effectiveness while managing such a large student population.

During the implementation of these course reforms, the introductory laboratories were updated with the aim of exposing students to a wide variety of physics concepts while streamlining the grading process. We will refer to this format as the "traditional lab." In the traditional lab, students solve 4-5 physics problems in a prelab worksheet, and work in groups in the classroom to follow detailed instructions in a "cookbook" style laboratory manual, filling their measurements and answers to questions into predetermined blank spaces provided in these sheets. Students are graded based on their ability to conform to the laboratory manual instructions and achieve the expected experimental results.

A typical traditional lab includes 3-4 distinct experiments using Microcomputer Based Laboratory (MBL) technology. Experiments are used to reiterate concepts covered previously in the course. Laboratory sessions meet once a week for two hour periods for nine weeks of the semester. A graduate student instructor supervises the laboratory and 
grades the prelab and lab handouts, providing written feedback as necessary.

Based on emerging research, student attitudes, and practical constraints, we have developed and piloted a new laboratory format, which we will refer to as the "IOLab hybrid lab," so called because it uses the Interactive Online Laboratory (IOLab) system - consisting of measurement equipment and a software interface - as the primary datacollection tool both in and out of the classroom [4]. The aim of this new format is to engage students in the practices of scientific thinking, particularly to develop their abilities to approach new problems and to evaluate new information. We chose these goals to align with the skills expected of engineering students entering their upper-level courses and the workplace [5].

In this new format, students complete two or three short activities in an online prelab assignment, and work in groups in the classroom on design tasks for which minimal written instruction is given. Prelab and classroom activities consisted of tasks and prompts designed to keep students feeling challenged to be creative in their experimental design and to build on the material they learned in class. Novel solutions to problems were praised by the instructor, and students were encouraged to make informed statements about their data rather than trying to obtain a "correct" value.

The prelab component of the IOLab hybrid lab was incorporated to combat limited classroom time and provide students with timely feedback on their laboratory preparation. Prelab activities were delivered online, and often included simple experiments using the IOLab equipment to observe some new behavior, design a measurement technique, or practice a new scientific skill. Students answered open-ended questions in free-response text boxes, and shared the data they collected in the prelab experiments with their instructor through an online repository system. Prelab assignments were due in advance of the laboratory class meeting, and students received individual email feedback from their instructor prior to class.

A typical IOLab hybrid lab meeting has one experiment which students design, perform, and record in an informal lab report that is turned in at the end of the class meeting. Classroom experiments were structured as observation, testing, or application experiments, with prompts similar to those described by Etkina, Murthy, and Zou in [6], and were graded using ISLE rubrics [7]. Use of the IOLab system at home and in the classroom made it easy for students to know the function and limitations of their data acquisition equipment. All other materials that were needed to do a given experiment were provided to the students, but it was up to them to decide which equipment and methods to use.

\section{Example of Prelab Activity}

Attach the spring to your force probe. Using your finger, apply a few quick horizontal impulses of varying strength on the end of the spring so that the IOLab remote rolls in the $+y$ direction each time.

1. Which sensors can you use to record information about the action described above?

2. Push on the IOLab as described above and share your data with your instructor.

3. What are some things you can find out using this data? List a few.

4. Choose one of the items from the list you just made and actually find that thing out. Describe what you did and what you found.

\section{METHODS}

Three sections of the calculus-based introductory mechanics course (Physics 211) were selected randomly to pilot the IOLab hybrid lab format in the spring 2016 semester. The remaining sections of the course used the traditional materials. Two of the IOLab hybrid lab sections were taught by the graduate student author of this paper, who has several semesters' teaching experience in this and other introductory physics courses. The remaining IOLab hybrid lab section was taught by a professor who, while not involved in PER, was one of the authors of the traditional laboratory curriculum used by the rest of the class. The new laboratory format met nine times through the duration of the semester, plus an additional time for a laboratory practical exam.

The traditional laboratory sections were taught by graduate students with varying levels of teaching experience. Labs in this format met nine times through the duration of the semester with no laboratory practical exam component, except for two lab sections which were selected by the researchers to act as a control for this study. Students in the control sections participated in the laboratory practical exam in place of the final laboratory meeting. These two sections were specifically chosen by the researchers to minimize differences in student population and quality of instructor between the traditional and IOLab hybrid lab sections being studied. The control sections were taught by an enthusiastic, highly-experienced graduate student, and met concurrently with the graduate student-taught IOLab hybrid lab sections.

Traditional and IOLab hybrid lab sections were the same size (enrollment of up to 33 students); however students in the traditional lab sections worked in groups of 4-5, while students in the IOLab hybrid lab sections worked in groups of 3-4. All other aspects of the course - including lecture, recitation, homework, and exams - were the same regardless of the student's laboratory experience.

\section{A. Measures}

Based on previous PER work on laboratories, and our own assessment of student experiences in the traditional laboratories at UIUC, we implemented measures to understand the effect of the new labs on student attitudes, 


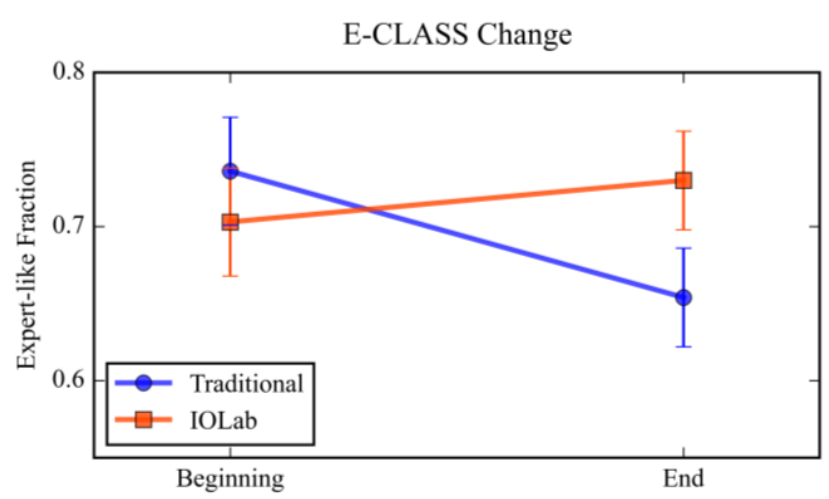

FIG 1. Fraction of expert-like responses on E-CLASS surveys administered at the beginning and end of the semester. The central values represent averages over all students and all statements on the survey, and the error bars represent one standard error of the mean for all statements on the survey.

ability to solve traditional exam problems, and scientific skills and behaviors. Measures of expert-like attitudes and exam performance were implemented for the entire course population. Measures of scientific skills and behaviors and student perception of learning were implemented during the laboratory practical exam to all students in the IOLab hybrid lab and to two sections of students from the traditional lab, as described previously.

We were interested in looking at two different aspects of student attitudes: their attitudes toward scientific research and their attitudes about their experience in our classroom. To probe the former, the Colorado Learning Attitudes about Science Survey for experimental physics (E-CLASS) [8] was administered online to all students in the course. Students' expert-like attitudes about experimental physics were measured during the first week and the final week of the semester.

To probe the latter, anonymous surveys measuring student perception of their laboratory classroom experience were administered on paper during the laboratory practical exam, using Likert-scale questions inspired by the Student Assessment of their Learning Gains (SALG) instrument [9]. Participation in this survey was voluntary.

The laboratory practical exam was developed to assess students' abilities to achieve the goals set out by the instructors for the IOLab hybrid laboratory. We are in the process of coding and analyzing students' written work on the laboratory practical exam, as well as coding video recordings of the different groups as they worked on experimental tasks. These data have not yet been fully analyzed, and will be presented in a future publication.

\section{RESULTS}

\section{A. Student Expert-like Attitudes}

The E-CLASS was administered online to the entire course on a voluntary basis. The overall expert-like responses from the IOLab hybrid lab students $(\mathrm{N}=61)$ and the traditional lab students $(\mathrm{N}=33)$ on the pre- and post-semester surveys is shown in Fig. 1. When we consider the mean over all items in the survey, results show no evidence of a pre-semester difference in overall expert-like attitudes between the two groups. The end of semester data shows no evidence of a change in expert-like attitudes from the IOLab hybrid lab students (pre: $70.3 \pm 3.5 \%$, post: $73.0 \pm 3.2 \%$ ). Students enrolled in the traditional laboratory displayed a decrease in expert-like attitudes at the end of the semester (pre: $73.6 \pm 3.5 \%$, post: $65.4 \pm 3.2 \%$ ). This result is not unexpected, since the traditional laboratory structure promotes habits that are inconsistent with expert-like attitudes measured in the E-CLASS. However, the very low response rate from the traditional students (3\% of the student population) may introduce selection bias, so that their population is not completely represented in these results.

\section{B. Student Test Scores}

Student scores on midterm exams and the final exam are shown in Table 1. Analysis of overall student scores on these exams reveals no measurable difference between students in the IOLab hybrid lab sections and students enrolled in the traditional lab sections $(\mathrm{p}=.56, \mathrm{p}=.97, \mathrm{p}=.98$, and $\mathrm{p}=.64$ for midterms 1, 2, and 3 , and the final exam, respectively).This result indicates that the choice to cover fewer concepts in the IOLab hybrid lab format did not put students at a disadvantage in the course.

\section{Student Perception of Learning}

The laboratory practical survey contained five questions to measure students' perception of the effect of the laboratory on their learning, and an additional question asking students to indicate how interesting they found the lab. Students were asked to indicate the extent to which a statement about their experience was true, with 1 meaning "the least" and 7 meaning "the most." Data showing student responses to these questions (IOLab N = 82 and Traditional $\mathrm{N}=47$ ),

TABLE I. Exam scores for the IOLab hybrid group $(\mathrm{N}=88)$ and overall course population $(\mathrm{N}=1010)$

\begin{tabular}{ccc}
\hline \hline Group & $\begin{array}{c}\text { Combined } \\
\text { midterm score }\end{array}$ & $\begin{array}{c}\text { Final exam } \\
\text { score }\end{array}$ \\
\hline IOLab hybrid & $77.99 \pm 2.63$ & $73.45 \pm 1.65$ \\
Traditional & $77.62 \pm 0.83$ & $74.16 \pm 0.52$ \\
\hline \hline
\end{tabular}




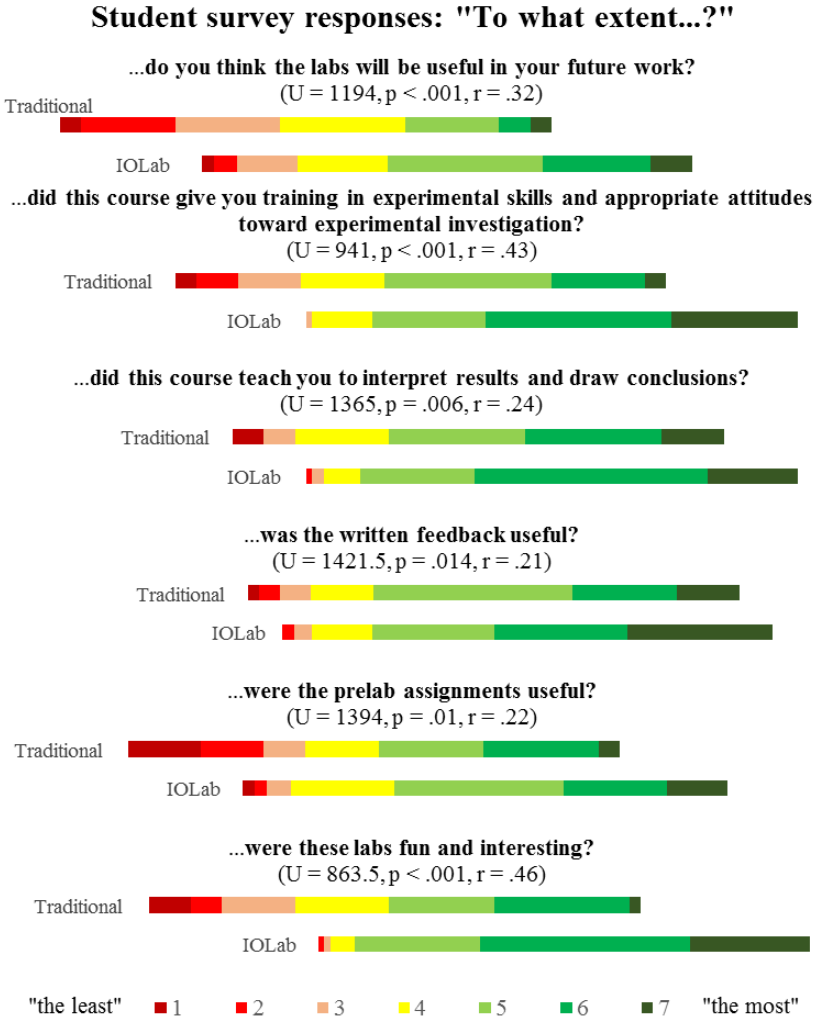

FIG 2. Stacked bar plots showing student responses to surveys administered during the laboratory practical exam. Mann-Whitney U test results are shown under the question text. IOLab Med. $=5$ and Traditional Med. $=4$ for items 1 and 5, IOLab Med. $=6$ and Traditional Med. $=5$ for all other survey items.

including the results of analysis using a Mann-Whitney $\mathrm{U}$ test is shown in Fig. 2. Looking at student responses, we observe that both populations were generally positive about their laboratory experience. The IOLab group consistently rated their experience with a median value one point higher.

[1] C. Wieman and N. G. Holmes, Am. J. Phys. 83, 972 (2015).

[2] E. Etkina and A. Van Heuvelen, Research-Based Reform of University Physics, edited by E.F. Redish, P. J. Cooney (American Association of Physics Teachers, College Park, MD 2007), Reviews in PER Vol. 1.

[3] D. K. Campbell, C. M. Elliot, and G. E. Gladding, Forum on Education Newsletter of the American Physical Society. (Summer), 9-11 (1997).

[4] More information on the IOLab system can be found at http://iolab.science

\section{CONCLUSIONS}

The new IOLab hybrid lab format at the University of Illinois aims to challenge engineering students while engaging them in scientific skills practices. After completion of a one-semester pilot of this format, we found that students were exceptionally receptive to this new format.

Further analysis is in process at this time to determine the effect of this laboratory instruction on students' scientific abilities and behaviors. Based on initial work and classroom observations, we anticipate promising results from analysis of student written work and video recordings of laboratory sessions.

Subsequent iterations of this pilot will include development of instructor training and a streamlined prelab feedback process, which will make it possible to scale this reform to the entire introductory mechanics course. Following the result from this first semester pilot, we are confident that, as we move forward to a second semester iteration of this new laboratory format in Physics 211, we will be able to continue to engage our students in laboratory experiences that are both challenging and rewarding.

\section{ACKNOWLEDGEMENTS}

The authors of this paper would like to acknowledge Eugenia Etkina for providing feedback and suggestions during development of the laboratory assignments and assessment tools. The E-CLASS team for administering the E-CLASS survey. Kathryn Woessner for analysis work on the laboratory practical survey.

This research is supported by the National Science Foundation TUES program under Grant No. 1122534.

[5] L. Feisel and A. Rosa, J. Eng. Educ. 94, 1 (2005).

[6] E. Etkina, S. Murthy, and X. Zou, Am. J. Phys. 74, 979 (2006).

[7] The ISLE rubrics can be found at https://sites.google.com/site/scientificabilities/rubrics

[8] B. M. Zwickl, T. Hirokawa, N. Finkelstein, and H.J. Lewandowski, Phys. Rev. ST Phys. Educ. Res. 10, $\underline{010120(2014)}$.

[9] E. Seymour, D. Wiese, A. Hunter, and S.M. Daffinrud, Paper presented at the National Meeting of the American Chemical Society (San Francisco, CA, $\underline{2000)}$. 